\title{
The concept of Laser Spot Thermography test rig with real-time data processing
}

\author{
J Roemer* \\ AGH University of Science and Technology, Cracow, Poland
}

\begin{abstract}
The paper shows the concept of Laser Spot Thermography test rig with real time data processing. Laser Spot Thermography is a promising method for non-destructive testing applications especially, when fully non-contact procedure is required. Unfortunately, the procedure as of now is hard to apply, due to technical difficulties related with data processing. Laser thermography test procedure involves recording of multiple sequences (even hundreds), which have to be processed and evaluated as a whole. The industry requires quick and precise results, which is hard to achieve with the amount of output data from the currently known test procedure. The paper gives a proposal for improving the laser thermography test procedure through data parameterization (curve fitting) for infrared sequences. The data parameterization allows for reduction of output data size, in particular temporary data storage in RAM. The reduction of data size leads to faster real time processing.
\end{abstract}

\section{LASER THERMOGRAPHY - STATE OF THE ART}

Thermography is a technique that allows for non-contact temperature imaging. Thermography is based on the recording of electromagnetic radiation emitted by each body at a temperature greater than absolute zero. The amount of radiated energy is non-linearly dependent on the bodies' temperature. This relationship and the knowledge of the object emissivity allows for precise temperature assessment of the tested body. Thermography is applied in the fields of mechanical and electrical engineering, medicine, civil engineering, material sciences, manufacturing and so on. Furthermore, thermography can be used as supplementary method for other diagnostic techniques like e.g. ultrasound testing or x-ray. Thermography also increasingly supports night vision systems widely used in the police force and military.

Thermography, in the context of non-destructive testing, can be divided into two groups: passive and active. Passive thermography is based on the imaging of objects (e.g. engines, gears, electrical equipment) during their normal operation [1-8]. Detection of the damage in those objects is based on searching for the presence of unnatural temperature changes. Those changes may be the evidence of a malfunction. Active thermography (e.g. laser thermography) requires external energy delivered into the tested object [9-11]. During testing, the body is thrown out of its thermodynamic equilibrium (present in normal operation) by an addition of external energy. The object is observed in the cycle of being forced out of equilibrium to going back. The damage detection is performed during this cycle by imaging and analyzing the thermal signature of the tested object. Any deviations from the expected trajectory of the system behavior may indicate a change in its physical parameters (assuming that the

$\overline{{ }^{*} \text { Corresponding Author: jroemer@agh.edu.pl }}$ 
measurement conditions are maintained during testing). The active thermography method, therefore, requires prior knowledge of the physical properties of the system - in particular, the shape of the expected trajectory defining the tested objects' behavior due to the introduced additional energy. System features such as objects' isotropy properties allow for a significant simplification of the procedure for determining the said trajectory [12-18].

Active thermography studies can be divided by the type of excitation. Each type of excitation defines the measurement procedure and the type of data processing techniques. Figure 1 shows the techniques most commonly used in practice.

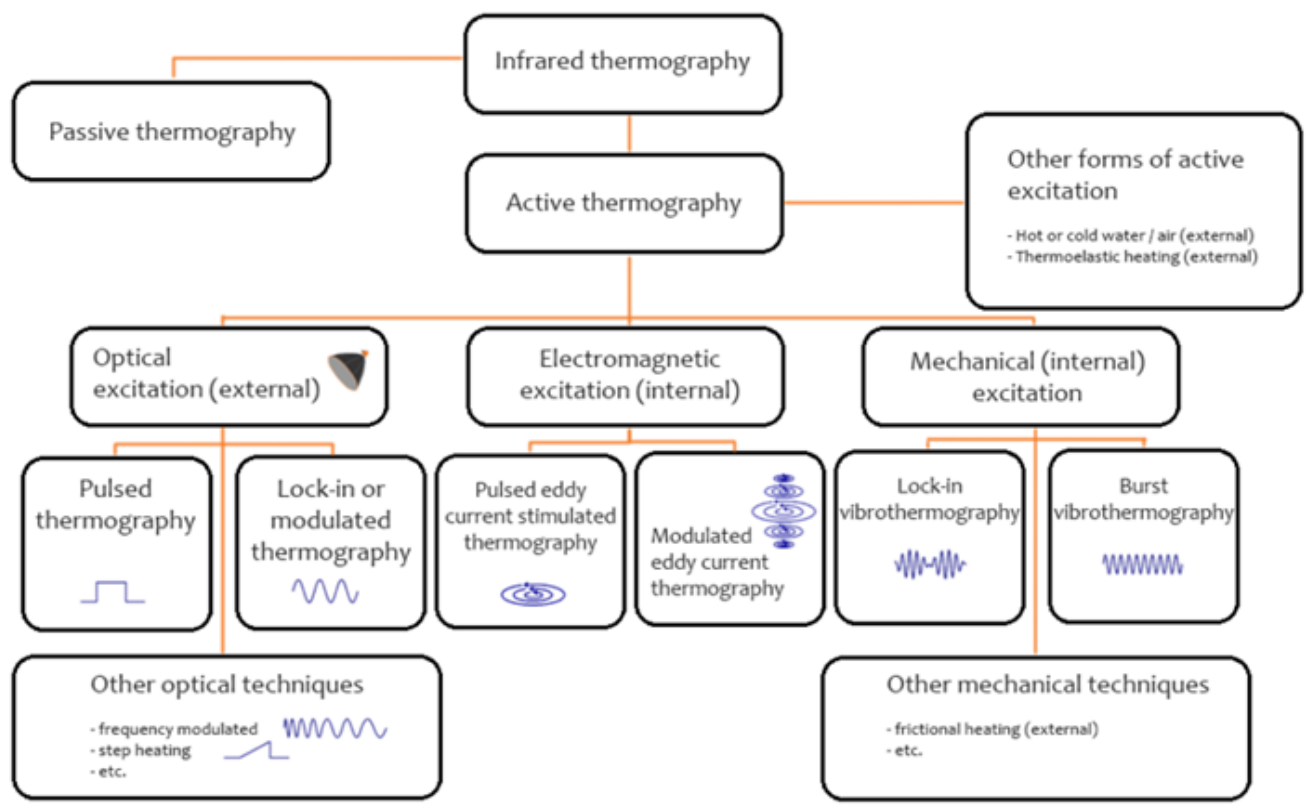

Fig 1. Active thermography - most commonly used techniques [18]

One of the newer and more interesting techniques using optical excitation is laser thermography. In the laser thermography procedure, the energy is delivered into the sample via laser beam. The delivered heat produces thermal wave propagating into the tested object. Every changes in the material conductivity, introduced e.g. by flaws, change the propagating thermal wave path. The thermal wave shape is determined on the basis of dynamically changing the examined structure surface temperature. The defect detection is based on the interpretation of the thermal wave propagation path. The system of laser spot thermography with the measurement procedure is shown on Figure 2.

The only interaction between laser, IR camera and the tested object is provided by the electromagnetic waves. Therefore, in a macroscopic world, laser thermography can be recognized as a non-contact technique. Both IR cameras and lasers can be used remotely (e.g. mounted on a robot) significantly reducing the human factor. Eliminating the human error creates advantage especially in applications where sterile, precise and repeatable conditions are necessary. 


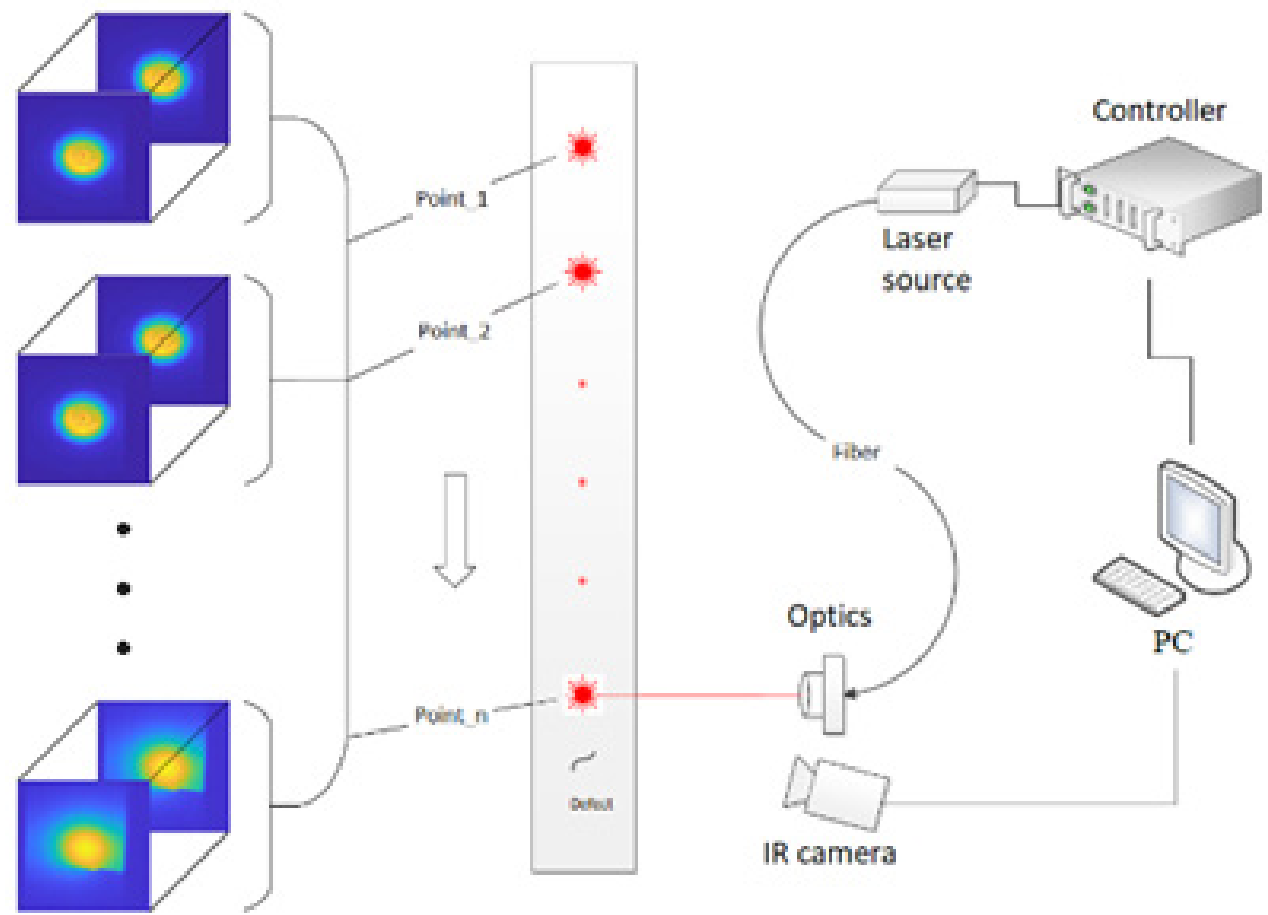

Fig 2. Laser thermography - setup and measurement procedure

\section{PROBLEM DEFINITION}

The literature [19 - 27] presents many case studies proving laser thermography effectiveness. Cracks, delamination, inclusions and porosity of the material can be detected and evaluated in an absolute non-contact and non-destructive manner. There are many successful examples of testing composites, electronics, 3D printed materials with the use of thermography. Unfortunately, most ideas, although initially positively verified, are only viable in the laboratory environment, and not in the industry. The reason for that can be found in data processing stage of the laser thermography testing process. In 'classical' pulse thermography with excitation provided by (e.g. halogen) lamps, each recorded sequence is treated as a standalone measurement. The critical part, which is the measurement interpretation, is realized by a well-trained technician. In that sense, the thermography procedure is similar to the procedures like X-rays or ultrasound testing.

The specific procedure of the laser spot thermography is based on multiple singular measurements, carried out point by point through the whole or interesting part of the sample. However, the assessment of the particular sample can be made based on the records performed for all measurement points at the same time. The natural solution for this problem would be a merger of all recorded samples into one big sequence. Technician could easily interpret sequence obtained that way, mainly in the same way as in the 'classic' pulse thermography systems. 


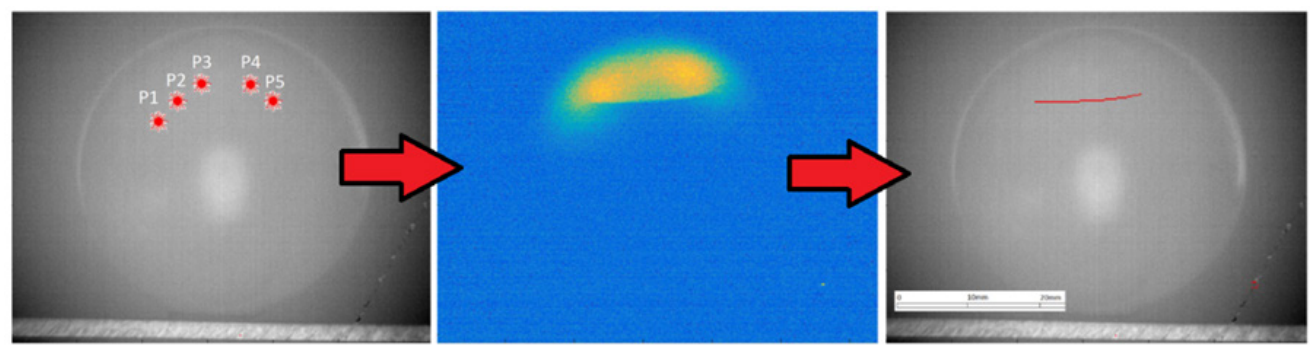

Fig 3. Laser thermography - sequence merging and crack detection [28]

Presented method seems to resolve aforementioned problems and allows well-trained technician for easy and reliable assessment of laser thermography scans. Unfortunately, this approach induces several another issues related with technical requirements for the test rig. In the example shown in Figure 3 sequences recorded for five measurement points were collected, stored, and processed. The singular sequence of $1 \mathrm{~s}$ with $640 \times 512$ spatial resolution and $20 \mathrm{~Hz}$ frame rate gives about $144 \mathrm{MB}$ of raw data. That means the scan of a sample, merged from five measurement points has about 0.7 GB. Presented example was just a proof of concept for laser thermography effectiveness for crack detection. The real-world scenario measurement procedure would require about 100 singular measurements for a scan. This would bring about $14 \mathrm{~GB}$ raw data, which has to be processed in real time considering time demand applications. This requirement is unlikely to fulfill in test case of a PC based test rig. Therefore, there is a strong need for developing alternative methods for data processing.

\section{THE CONCEPT}

Thermographic testing creates relatively large amount of raw data from the measurement of the tested object cooling stage. For a single record that is not a problem, however for multiple measurements it becomes an issue. The tested sample needs to be examined point by point to create a single scan of the sample. For multiple measurements each point can be represented by a sequence of raw data in time. The sequence indirectly holds information on the curve shape of the wave propagation through the object during its cooling phase. The conceptual proposition is raw data reduction by conversion into a parametric form. In each scan the points can be represented by a parameter, which can vary in the whole sequence. Appropriately created parametric form would preserve most important features of the thermal wave created during the thermographic measurement. At the same time parametric representation will significantly reduce the measurement data memory size.

Figure 4 presents the proposed measurement procedure consisting of several steps. In first step the IR sequence is recorded identically as it is performed for laser thermography. At this stage the data is not stored on HDD but remains in RAM memory of the camera controller or the frame grabber. The next step is the parametric matrix integration, which involves frame sizing (according to the region of interest), filtering, background subtraction and all other necessary preprocessing methods. During the third step the measurement sequence parametric form is created based on an analytical model. At this point data is still in RAM memory close to the CPU, therefore all computations can be performed at a very short time. The procedure's output is a set of several parameters describing the dynamic nature of the thermal wave created in a single laser thermography measurement. 
SINGLE MEASUREMENT

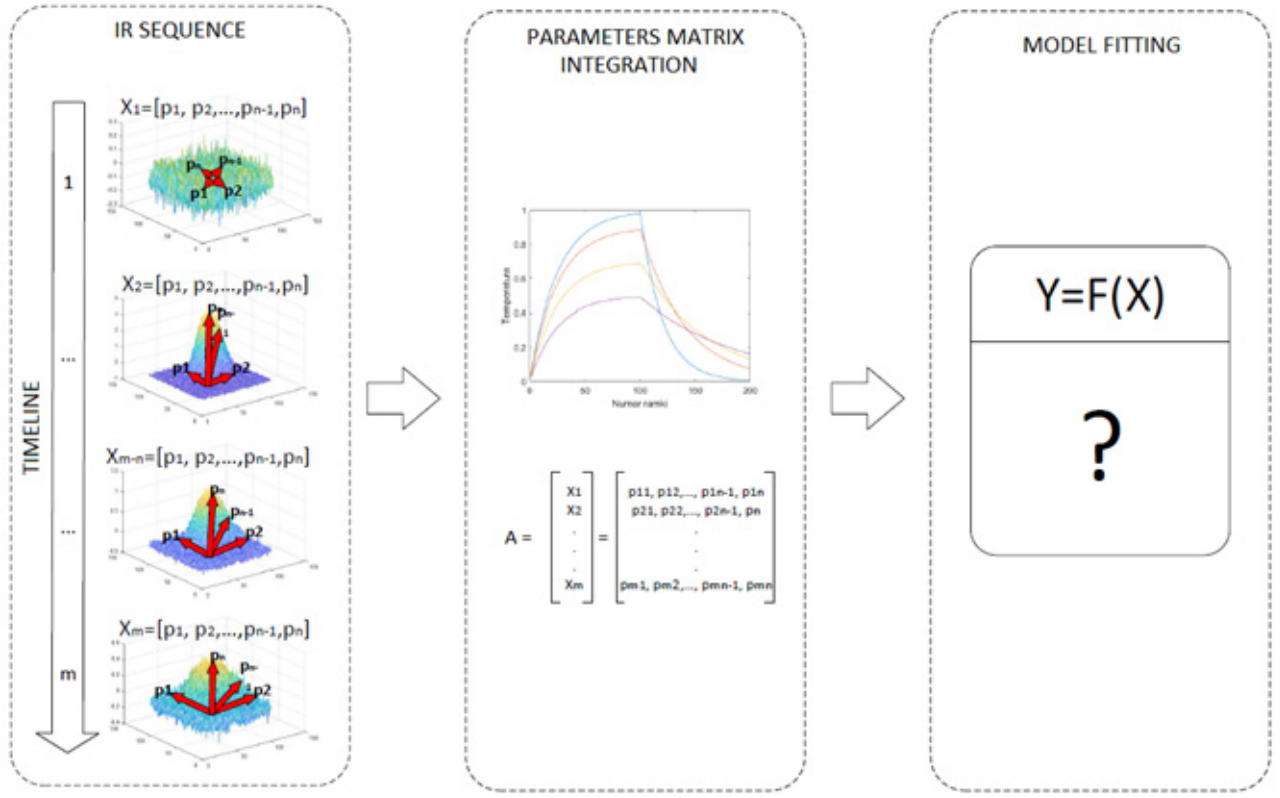

Fig 4. Infrared sequence transformation

Figure 5 shows the procedure for creating 3D scans based on subsequent individual measurements. The scan contains data for each measured point sequence in time. The 3D scan is created in the offline mode after the entire measurement has been carried out. The raw data parameterization and composition of the 3D scan offers not only reduction in data size, but also data readability and easier post-processing. In this context, the pre-processed measurement data can be easily shared online, transferred faster between PC's or even smartphones, and the data simplified form allows for straightforward implementation e.g. of a machine learning algorithm or data post-processing visualization. The data size reduction has the most impact in historical data storage, which is crucial for quality control applications.

Figure 6 shows a concept scheme of the laser thermography test system. The test system consist of seven modules: Controller (main module), laser, IR (camera), positioner (e.g. robot arm), data processing (real time), Graphical User Interface (on PC or smartphone) and Data analysis (locally or remotely on a server). The modular structure of the system gives flexibility in implementing laser thermography for different case scenarios. 
3D SCAN
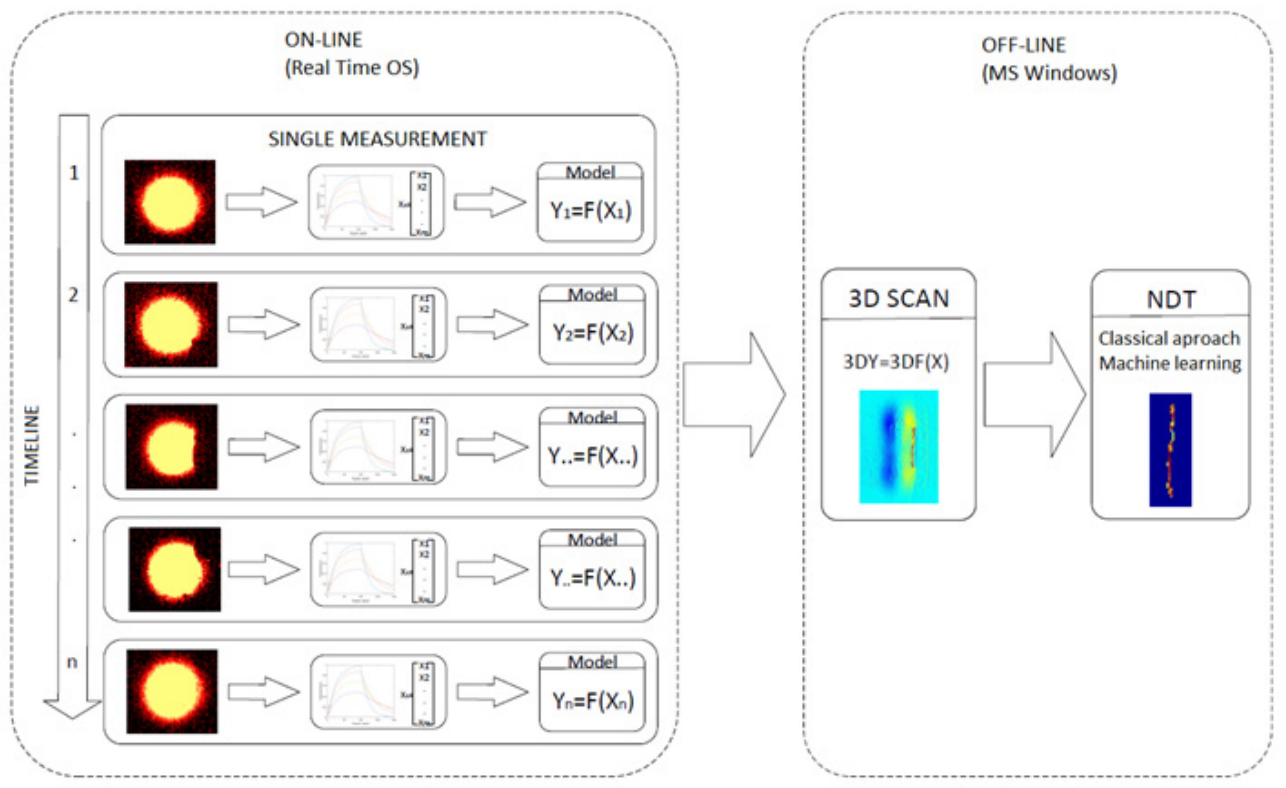

Fig 5. 3D scan forming and interpreting

\section{MEASUREMENT AND CONTROL SYSTEM}

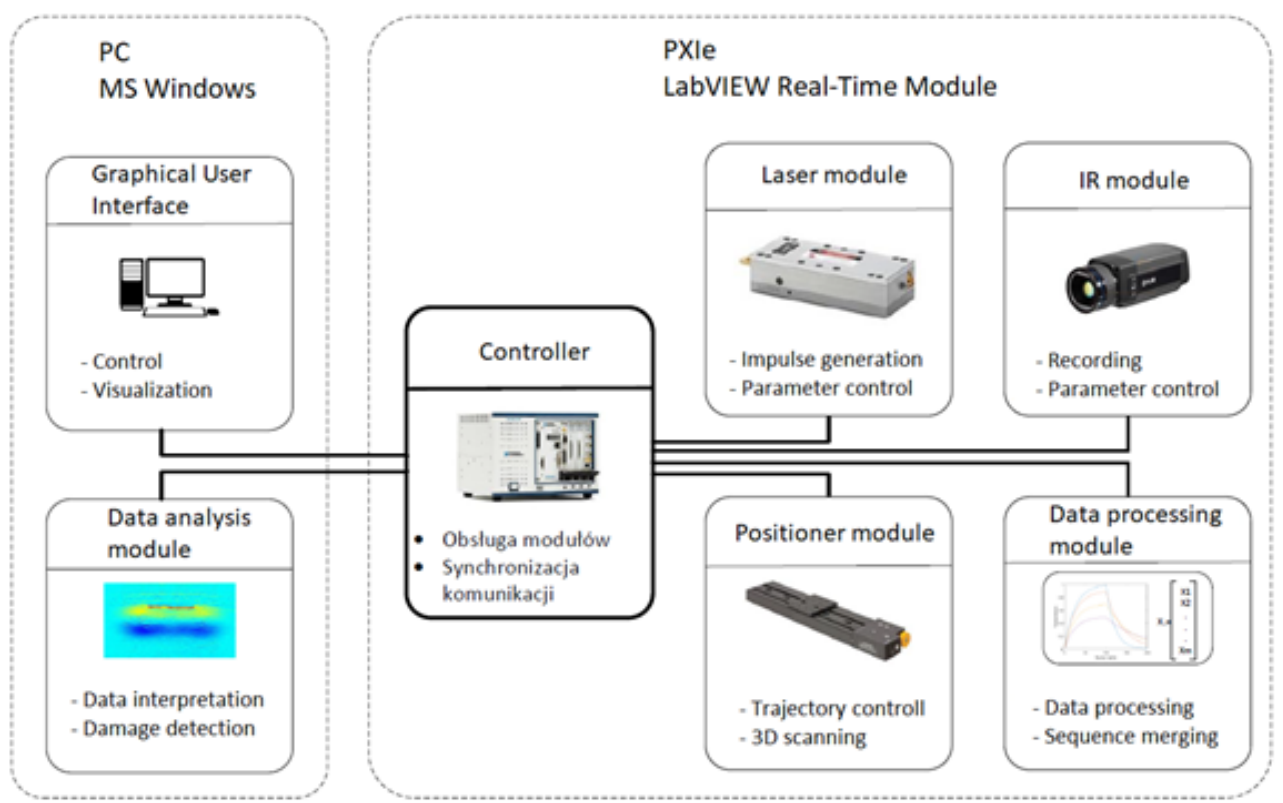

Fig 6. Concept of the laser thermography test system 


\section{SUMMARY}

The paper presents a concept of Laser Spot Thermography test rig and a technique for measurement data parameterization. The proposed technique allows for online pre-processing of recorded data, while forming a 3D scan structure as an output. The 3D scan holds information about the sample scans at each measurement point as vectors of several parameters. Those vectors store not the measurement raw data, but the data parameter according to the analytical model describing the thermal characteristic of a particular tested material. The parametrization offers substantial reduction of data memory size in the order of several magnitudes. Packaging data in the form of a 3D scan allows for easier data postprocessing (e.g. simple input to a machine learning algorithm, more readable data format), faster data transfer due to size reduction, and smaller data storage size requirements (especially necessary for quality control over long periods of time). The real test rig built at AGH UST Kraków, Poland will be presented in the near future in upcoming publications.

\section{ACKNOWLEDGEMENTS}

This work has been supported by the AGH University, WIMiR, research grant no. 16.16.130.942

\section{REFERENCES}

[1] MALDAGUE, Xavier, et al. Theory and practice of infrared technology for nondestructive testing. 2001

[2] STANGE, Even, et al. Multiphysics Study of Tensile Testing using Infrared thermography. International Journal of Multiphysics, 2019, 13(2): p.191-202.

[3] STANGE, Even, et al. Qualitative visualization of the development of stresses through infrared thermography. Vestnik of MSTU, 2019, 22(4): p. 503 - 507.

[4] AHMAD, Tanveer, et al. Study of the required thermal insulation (IREQ) of clothing using infrared imaging. International Journal of Multiphysics, 2017, 11(4): p. 413-426.

[5] RASHID, Taimur, et al. Determination of Thermal Properties of Fresh Water and Sea Water Ice using Multiphysics Analysis. International Journal of Multiphysics, 2016; 10(3): p. 277 - 291.

[6] KHAWAJA, Hassan et al. Multiphysics Simulation of Infrared Signature of an Ice Cube. International Journal of Multiphysics, 2016, 10(3): p. 291 - 302.

[7] AHMAD, Tanveer, et al. Study of Wind Chill Factor using Infrared Imaging. International Journal of Multiphysics, 2016, 10(3): p. 325 - 341.

[8] RASHID, Taimur et al. Review of marine icing and anti-/de-icing systems. Review of Marine Engineering \& Technology, 2016, 15(2): p. 79 - 87.

[9] RASHID, Taimur, et al. Measuring Thickness of Marine Ice Using IR Thermography. Cold Regions Science and Technology, 2019, 158: p. 221-229.

[10]T. Uhl, M. Szwedo, Ł. Pieczonka, J. Roemer, P. Hellstein. "Damage detection in composite structures using active thermography - case studies". European congress and exhibition on Advanced materials and processes. Warszawa, Polska, 2015

[11] PIECZONKA, Łukasz; SZWEDO, Mariusz. Vibrothermography. In T. Stepinski, T. Uhl, \& W. J. Staszewski (Eds.), Advanced Structural Damage Detection: From Theory to Engineering Applications (pp. 233-261). Wiley. 2013.

[12]ROEMER, Jakub; PIECZONKA, Łukasz; UHL, Tadeusz. Laser spot thermography of welded joints. Diagnostyka, 2014, 15 
[13]ROEMER, Jakub, et al. Thermography of metallic and composite structures-review of applications. Int. Work. Smart Mater. Struct. SHM, 2013, 18.11.

[14]ROEMER, Jakub; UHL, Tadeusz; PIECZONKA, L. Laser spot thermography for crack detection in aluminum structures. In: 7th International Symposium on NDT in Aerospace. 2015. p. 1-7.

[15] PIECZONKA, L., et al. Modelling and numerical simulations of vibrothermography for impact damage detection in composites structures. Structural Control and Health Monitoring, 2013, 20.4: 626-638.

[16]PIECZONKA, Łukasz; SZWEDO, Mariusz; UHL, Tadeusz. Investigation of the effectiveness of different thermographic testing modalities in damage detection. In: Key Engineering Materials. Trans Tech Publications, 2013. p. 349-356.

[17] SZWEDO, M. Pieczonka Ł., Uhl T., 2011, Image processing technique for vibrothermographic field tests. In: Proceedings of the 8th International Workshop on Structural Health Monitoring. 2011. p. 13-15.

[18] Workswell s.r.o. (www.workswell-thermal-camera.com)

[19]CERNIGLIA, Donatella; MONTINARO, Nicola. Defect Detection in Additively Manufactured Components: Laser Ultrasound and Laser Thermography Comparison. Procedia Structural Integrity, 2018, 8: 154-162.

[20]ZHANG, Hai, et al. An experimental and analytical study of micro-laser line thermography on micro-sized flaws in stitched carbon fiber reinforced polymer composites. Composites Science and Technology, 2016, 126: 17-26.

[21] MEZGHANI, S., et al. Evaluation of paint coating thickness variations based on pulsed Infrared thermography laser technique. Infrared Physics \& Technology, 2016, 76: 393401.

[22] KEO, Sam Ang, et al. Defect detection in CFRP by infrared thermography with CO2 Laser excitation compared to conventional lock-in infrared thermography. Composites Part B: Engineering, 2015, 69: 1-5.

[23] AN, Yun-Kyu, et al. Line laser lock-in thermography for instantaneous imaging of cracks in semiconductor chips. Optics and Lasers in Engineering, 2015, 73: 128-136.

[24]BEDOYA, A., et al. Measurement of in-plane thermal diffusivity of solids moving at constant velocity using laser spot infrared thermography. Measurement, 2019, 134: 519526.

[25] HWANG, Soonkyu; AN, Yun-Kyu; SOHN, Hoon. Continuous Line Laser Thermography for Damage Imaging of Rotating Wind Turbine Blades. Procedia Engineering, 2017, 188: 225-232.

[26] MONTINARO, Nicola; CERNIGLIA, Donatella; PITARRESI, Giuseppe. Defect detection in additively manufactured titanium prosthesis by flying laser scanning thermography. Procedia Structural Integrity, 2018, 12: 165-172.

[27]MONTINARO, N.; CERNIGLIA, D.; PITARRESI, G. Flying laser spot thermography technique for the NDE of fibre metal laminates disbonds. CompositeStructures, 2017, 171: 63-76.

[28] ROEMER, J., et al. Nondestructive Testing of Ceramic Hip Joint Implants with Laser Spot Thermography. Archives of Metallurgy and Materials, 2017, 62.4: 2133-2139. 